\title{
奥氏体/ 马氏体异相界面的电子密度
}

\author{
刘志林 孙振国 李志林 \\ (辽宁.上学院材料上程系, 触州 121001)
}

\section{关键词余氏理论 程氏理论 界面}

复相合金与复合材料中都存在着异相界面. 改善基体与第二相界面的工作已成为材料研 究中的重要课题. 近年来在复合材料中提出的强界面结合及弱界面结合模型、基体与增强体 相匹配的强化机理等在复相合金中也同样适用。这些研究涉及界面物理及界面化学, 也包括 界面的原子结构及电子状态. 程开甲先生认为通常的 TFD 模型在材料设计、电子输运现象 等实用领域中不完善, 似乎是在处理原子条件中, 特别是在固体中原子的边界条件处理上: 有 一些错误, 正确的边界条件是存在的, 只是一直未找到 ${ }^{[1 !}$. 程开甲指出, 原子的边界条件 只是电子密度要连续, 因为这是量子力学所要求的波函数连续的条件 ${ }^{[1]}$. 按这一设想, 本文用 “余氏理论” ${ }^{22}$ 计算了奥氏体/马氏体 $(\mathrm{A} / \mathrm{M})$ 界面的共价电子分布, 一级近似下其平均共价电子 密度 (简称“电子密度”) 连续, 与“程氏理论”提出的原子边界条件相符. 这一结果不仅提出了 异相界面电子密度计算的一种方法, 也证实了文献[1] 提出的 “改进的 TFD 模型可以作为研究 湖体电子理论的探针”。

\section{A/M 异相界面电子密度的计算}

与氏体相变的晶体学特点是新相和母相之间存在一定的位问关系, 转变后两相的位相关 系仍保持着. 无论钢的含碳量如何均有 (111), / (110) 的位们关系. 本文的 $\mathrm{A} / \mathrm{M}$ 异相界面 也5 密度计算选择了含 $\mathrm{C}$ 重量的 $0.8 \%$ 的 Fe-C 合金, 令 $\rho_{(\mathrm{hkl})}$ 为异相界面的电子密度,

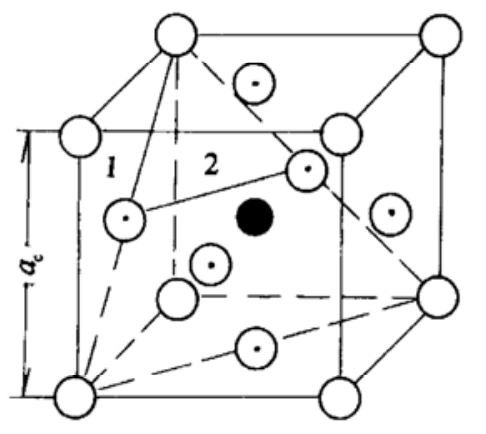

图 1 奥氏休含 $\mathrm{C}$ 晶胞的 (111), , 为 $\mathrm{Fe}^{\mathrm{c}}$, $\bullet$ 为 $\mathrm{Fe}^{\mathrm{r}},-$ 为 $\mathrm{C} .1$ 为 $\mathrm{D}_{\mathrm{nB}}^{\mathrm{rec}} \mathrm{Fe}^{\mathrm{r}}$, 2 为 $\mathrm{D}_{\mathrm{nc}}^{\mathrm{re}} \mathrm{re}^{\mathrm{r}} \cdot \mathrm{re}$

1995-01-13 收稿, 1995-05-02 收修政稿 $n_{\mathrm{c}}^{(\mathrm{mkl})}$ 为异相界面的共价电子总数 (即该面等效共价揵上 电子数月的总和), $S_{(\mathrm{hkl})}$ 为异相界面的面积, 则有

$$
\rho_{(\mathrm{hkl})}=n_{\mathrm{c}}^{(\mathrm{hkl})} / S_{(\mathrm{hkl)}} \text {. }
$$

\section{1 奥氏体 $(111)_{\gamma}$ 晶面的电子密度}

文献[3] 曾按文献[4] 的方法计算了含 C 重量 $0.80 \%$ 的奥氏体的价电子结构, 图 1 给出了奥氏体 (111) 晶面及 其面上的共价键络. 如果按文献[5], $\mathrm{C}-\mathrm{Fe}^{\mathrm{f}}$ 原子产生短程 偏聚, 奥氏体中 (111) 的衍射可不考虑 $\gamma-\mathrm{Fe}$ 唱胞 (111) 面的贡献, 丁是 (111) 的面积为

$$
S_{(111)_{i}}=\frac{\sqrt{3}}{2} a_{\mathrm{c}}^{2}=0.12285 \mathrm{~nm}^{2} .
$$


文献[4] 计算等效键的公式 $I_{\alpha}=I_{\mathrm{M}} \cdot I_{\mathrm{S}} \cdot I_{\mathrm{K}}$ 可计算分布在 $(111)_{\gamma}$ 上的等效键 ${ }^{[3]} D_{\mathrm{nB}}^{\mathrm{Fe}-} \mathrm{Fe}^{\mathrm{f}}$ 、 $D_{\mathrm{nC}}^{\mathrm{re}}-\mathrm{re}{ }^{\mathrm{f}}$ 的数目分别为 6 , 键上的共价电子数分别为 0.2361 和 0.2311 . 于是

\section{2 马氏体 $(\mathbf{1 1 0})_{\alpha}$ 晶面的电子密度}

$$
\begin{aligned}
& n_{\mathrm{c}}^{(111)_{\gamma}}=(0.2361+0.2311) \times 6=2.8032 \text {, }
\end{aligned}
$$

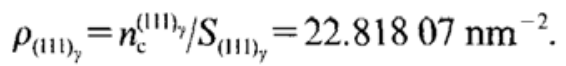

文献[6] 根据 Choo 和 Kaplow 的实验, 选取 C 原子和它的第一近邻 $\mathrm{Fe}_{\mathrm{I}}$ 原子、第二近邻 $\mathrm{Fe}_{\text {III }}$ 原子、第三近邻 $\mathrm{Fe}_{\mathrm{III}}$ 原子构成的含 $\mathrm{C}$ 结构单元计算了含 $\mathrm{C}$ 重量 $1.70 \%$ 的马体的价电 子结构. 文献[3] 也用这个模型计算过含 $\mathrm{C}$ 重量 $0.80 \%$ 的马氏体的价电子结构. 但这两例 计算都略去了 $\mathrm{Fe}_{1}$ 和 $\mathrm{Fe}_{\mathrm{III}}$ 原子间的共价键. 本文异相界面精细结构的分析仍用文献[6] 的模 型并考虑了 $\mathrm{Fe}_{1}-\mathrm{Fe}_{\mathrm{III}}$ 的共价键. 图 2 即为马氏体的含碳 结构单元. 图中绘出了它的 (110) 晶面及面上的共价键 分布. 在 (110) 面上各类等效键数的计算仍按文献[4] 的

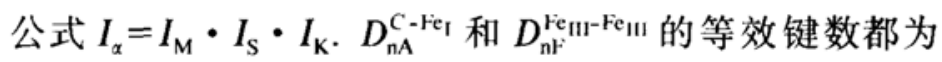

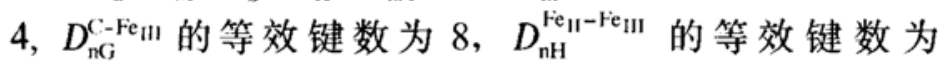
2. 含 $\mathrm{C}$ 重量 $0.80 \%$ 的马氏体含碳晶胞的价电子结构 见表 1. 由表 1 可查得 (110) 唱面上的 $D_{\mathrm{nA}}^{\mathrm{C}-\mathrm{Fe}}$ 键的共

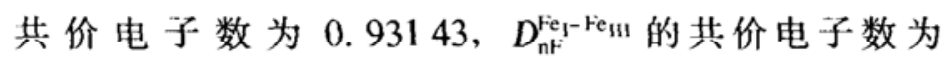
0.179 89. $D_{\mathrm{nG}}^{\mathrm{C}-\mathrm{Fe}} \mathrm{III}_{\text {II }}$ 的共价电子数为 $0.01024, D_{\mathrm{nB}}^{\mathrm{Fe}_{\mathrm{II}}-\mathrm{Fe}_{\mathrm{III}}}$ 的 共价电子数为 0.00785 .

如果认为与氏体中的 $\mathrm{C}-\mathrm{Fe}_{1}, \mathrm{C}-\mathrm{Fe}_{11}$ 也处于偏聚状 态, 图 2 中的 (110) 唱面也会扩展到足够大而使衍射中 不考虑马氏体中 $\alpha-\mathrm{Fe}$ (不含碳唱胞) 的 (110) 面对衍射

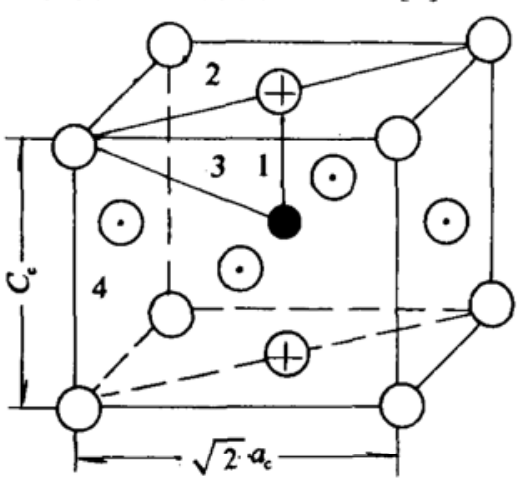

图 2 与氏体含碳结构单元的 (110) 的晶面

$\oplus$ 为 $\mathrm{Fe}_{\mathrm{l}},\left(\bullet\right.$ 为 $\mathrm{Fe}_{\mathrm{ll}}, O$ 为 $\mathrm{Fe}_{\mathrm{II}}, \bullet$ 为 C. 1

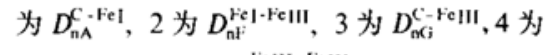
$D_{\mathrm{nH}}^{\mathrm{iel} I I+F e I I I}$ 的贡献. 于是

$$
S_{(110) \alpha}=2 a_{c} c_{\mathrm{c}}=2 \times 0.26963 \times 0.36927=0.19913 \mathrm{~nm}^{2},
$$

\begin{tabular}{|c|c|c|c|c|c|}
\hline$A_{\mathrm{c}}: 3.626$ & & $a_{0}: 0.28664 \mathrm{~nm}$ & $a_{\mathrm{c}}: 0.26963 \mathrm{~nm}$ & \multicolumn{2}{|c|}{$C_{\mathrm{c}}: 0.36927 \mathrm{~nm}$} \\
\hline $\mathrm{Fe}_{1}$ & & $\mathrm{Fe}_{\|}$ & $\mathrm{Fe}_{\mathrm{tII}}$ & \multicolumn{2}{|c|}{$\mathrm{C}$} \\
\hline$\sigma: A$ & & $\sigma: \mathbf{A}_{10}$ & $\sigma: \mathbf{A}_{9}$ & \multicolumn{2}{|c|}{$\sigma: \mathrm{A}_{6}$} \\
\hline$n_{\mathrm{c}}^{\prime \prime}: 4.20^{\prime}$ & & $n_{\mathrm{c}}^{10}: 3.9723$ & $n_{\mathrm{c}}^{9}: 3.7743$ & \multicolumn{2}{|c|}{$n_{\mathrm{c}}^{6}: 4.0000$} \\
\hline$R(I)^{\prime \prime}: 0$. & & $R(I)^{10}: 0.10919 \mathrm{~nm}$ & $R(I)^{9}: 0.11059 \mathrm{nr}$ & \multicolumn{2}{|c|}{$R(I)^{6}: 0.0763 \mathrm{~nm}$} \\
\hline \multicolumn{6}{|c|}{$\beta=0.7100$} \\
\hline 键名 & $I_{\alpha}$ & $D_{\mathrm{nx}}(\mathrm{nm})$ & $\widetilde{D}_{\mathrm{na}}(\mathrm{nm})$ & $n_{x}$ & $\Delta D_{n_{\alpha}}(\mathrm{nm})$ \\
\hline$D_{\mathrm{nA}}^{\mathrm{c}^{-}+\mathrm{c}_{1}}$ & 4 & $0.184 \quad 64$ & 0.18601 & 0.93143 & 0.00137 \\
\hline$D_{\mathrm{nB}}^{\mathrm{C}-\mathrm{rec}_{11}}$ & 8 & $0.190 \quad 66$ & 0.19203 & 0.80881 & 0.00137 \\
\hline$D_{\mathrm{nc}}^{+\mathrm{e} I 1-\mathrm{Fe}_{\mathrm{III}}}$ & 16 & 0.26541 & 0.26678 & 0.21781 & 0.00137 \\
\hline$D_{n \mathrm{D}}^{\mathrm{ref}-\mathrm{re}} \mathrm{e}_{1 \mathrm{I}}$ & 16 & $0.265 \quad 41$ & 0.26678 & 0.19711 & 0.00137 \\
\hline$D_{\mathrm{nF}}^{\text {Fell-kellI }}$ & 8 & 0.26963 & 0.27100 & 0.18144 & 0.00137 \\
\hline$D_{\mathrm{nF}}^{F i-F e l l}$ & 8 & 0.26963 & 0.27100 & 0.17989 & 0.00137 \\
\hline$D_{\mathrm{n} j}^{\mathrm{C}-\mathrm{re}} \mathrm{III}$ & 16 & 0.32679 & 0.32816 & 0.01024 & 0.00137 \\
\hline$D_{\mathrm{nH}}^{\mathrm{re} H I I-F \mathrm{c}} \| 1 \mathrm{I}$ & 2 & 0.36927 & 0.37064 & 0.00785 & 0.00137 \\
\hline$D_{\mathrm{nl}}^{\mathrm{Fe}}$ & 4 & $0.381 \quad 32$ & 0.38269 & 0.00531 & 0.00137 \\
\hline \multicolumn{2}{|c|}{$\sum I r=21.14606$} & \multicolumn{2}{|c|}{$\sum n_{\mathrm{c}}=20.1244$} & \multicolumn{2}{|c|}{$n_{\mathrm{c}}^{\mathrm{D}}=1.7881$} \\
\hline
\end{tabular}

表 1 含 $\mathrm{C}$ 重量的 $0.80 \%$ 的马氏体含碳晶胞的价电子结构 


$$
\begin{aligned}
& n_{\mathrm{c}}^{(110)_{x}}=(0.93143+0.17989) \times 4+0.01024 \times 8+0.00785 \times 2=4.54290 \text {, } \\
& \rho_{(110)_{\alpha}}=n_{\mathrm{c}}^{(110)_{\alpha} / S_{(110)_{\alpha}}}=22.81374 \mathrm{~nm}^{-2} \text {. }
\end{aligned}
$$

\section{$2 \mathbf{A} / \mathbf{M}$ 异相界面的电子结构}

由同类原子组成的固体如金属, 原子边界的电子结构是不难理解的. 由不同种原子组成 的固体如合金, 不同种类的原子将作为组元存在于合金相中. 相中各向异性的共价键把不同 种类的原子键合在一起, 原子的边界应该是相的边界. 本文的计算表明, $\mathrm{A} / \mathrm{M}$ 界面两侧的共 价电子密度几乎相等, 这正是程氏理论指出的 “固体中原子间的边界条件只是电子密度要连 续”的量子力学条件.

\section{3 讨论}

(1) 本文计算 $A / M$ 异相界面的电子密度采用的是 “偏聚模型” [3, 4, 6], 当采用 “平均晶胞模 型” ${ }^{[4]}$ 计算时也有同样的结果. 对 $\mathrm{A} / \mathrm{F}$ (奥氏体/铁素体)、 $\mathrm{F} / \mathrm{Fe}_{3} \mathrm{C}$ (铁素体/渗碳体) 等异相界面 所做的研究表明, 一级近似下这些界面的电子密度也是连续的. 计算结果揭示了余氏理论和 程氏理论可以在“异相界面平均共价电子密度连续”这一接合点上实现衔接.

(2) 文献[4] 已经给出了余氏理论计算中确定原子杂化状态的原则, 大量的计算和一些实用 分析表明按文献[4] 确定的原子状态不乏其准确性,但对不同的研究对象和不同的研究者也有 经验上的偏差．本文的计算表明，按文献[4] 的原则加上“固体中原子间的边界条件”可使余氏 理论中原子状态的确定更加准确. 这正是程氏理论预言的 “改进的 TFD 模型可以作为研究 固体电子理论的探针”的一个印证.

\section{参考 文 献}

1 犁开甲,程做玉. TFD 模型和余氏理论对材料设计的应用. 自然科学进展, 1993，3(5): 417

2 余瑞璜. 固体与分子经验电子理论. 科学通报, 1978, 23(4): 217

3 刘志林. 合金价电子结构与成分设计. 长春：吉林科学技术出版社, 1990. 26 123

4 张瑞林. 固体与分子经验电子理论. 长春：古林科学技术出版社, 1993. 236 316

5 Liu Zhilin. C-Me segregating theory in solid alloys. Chinese Science Bulletin, 1989, 34(23): 2011

6 张瑞林, 余瑞横. Fe- C 马氏体价电子结构分析. 金属学报, 1984, 20(4)：A279 